\title{
Hypertension - state of the art 2017
}

\author{
Author: Linsay McCallum
}

\section{Introduction}

Annually there are 9.4 million deaths worldwide attributed to hypertension, which remains the largest contributor to the global burden of disease and mortality. ${ }^{1}$ Hypertension is common, affecting one third of adults over the age of 18 years, with a projected increase in prevalence due to the growing population and ageing society. Despite increasing awareness and access to a number of antihypertensive drug classes, of those on treatment for hypertension only 30-40\% are controlled to current target. Controlling blood pressure (BP) with antihypertensive drugs is one of the most cost-effective methods of reducing premature cardiovascular morbidity and mortality. $^{2}$

The International Society of Hypertension (ISH), supported by the World Hypertension League and national societies, deemed May 2017 May Measurement Month (MMM). The ambitious goal of MMM was to screen 25 million people globally to highlight the need for increased BP screening, to identify and reduce the BPs of people found to have hypertension.

On the eve of World Hypertension Day 2017, the Royal College of Physicians (RCP) and the British and Irish Hypertension Society (BIHS) held the 'Hypertension - state of the Art 2017' conference bringing together a wealth of expert speakers to provide an update on recent advances in the understanding and management of hypertension.

\section{New evidence in 2017: how will it change practice?}

The optimum fourth-line antihypertensive agent was investigated in the PATHWAY-2 study with spironolactone emerging as the most effective agent compared with bisoprolol, doxazosin and placebo. ${ }^{3}$

Single pill combinations (SPCs) have been shown to be quicker and more effective in controlling BP than monotherapy or the combination of two single drugs with reduced side effects, improved patient adherence, improved cardiovascular protection and associated cost savings.

Worldwide, guidelines for the management of hypertension have stratified patients according to ethnicity; however, the evidence base for the optimum antihypertensive agent for each subgroup remains controversial. The CREOLE trial is

Author: clinical lecturer in clinical pharmacology, University of Glasgow, Glasgow, UK a multicentre, multinational, randomised study comparing three combinations of perindopril, amlodipine and hydrochlorthiazide in 700 hypertensive black African adults. CREOLE will complete and report in 2019 giving new insight into antihypertensive drug choice.

\section{BP targets: is higher or lower better?}

BP targets are controversial; the national and international societies are not in agreement as to what is the optimum BP target. The results of SPRINT (Systolic Blood Pressure Intervention Trial) - published in late 2015 and showing that a lower systolic BP target $(<120 \mathrm{mmHg})$ in patients at high risk of cardiovascular disease without diabetes is associated with a lower risk of fatal and non-fatal cardiovascular events - reignited the controversy. ${ }^{4}$ Previous studies have shown an association between systolic BP $<120 \mathrm{mmHg}$ and adverse outcomes, ${ }^{5,6}$ while a study of 1.25 million people using electronic health records reported no J-shaped association. ${ }^{7}$ The diastolic BP target is also important, with evidence suggesting the presence of a J-shaped relationship ${ }^{8,9}$ between diastolic BP and adverse outcomes, particularly in frail older patients or those with coronary artery disease - perhaps a consequence of lowered coronary perfusion pressure. $^{10}$

\section{Measuring blood pressure}

Approximately one-third of patients with hypertension perform home BP monitoring (HBPM). HBPM may be more useful than clinic BP for the diagnosis of hypertension, but ambulatory $\mathrm{BP}$ monitoring (ABPM) remains the gold standard. Elevated BP recordings in one or more modality (HBPM, ABPM, clinic BP) put the patient at a greater risk of death. ${ }^{11}$ HBPM has been used with active management in the TASMINH2 study, where patients self-titrated antihypertensive agents according to HBPM and pre-agreed protocols. There were more medication changes in the group who self-managed with $50-70 \%$ of patients up-titrating per protocol and no associated increase in adverse events. ${ }^{12}$ ABPM has advantages over HBPM in assessing BP variability and identifying masked hypertension. Ongoing studies are investigating which measurement of BP (24 hour, day or night recordings) is the most useful predictor of risk.

\section{Hypertension and heart failure}

The presence of uncontrolled hypertension is strongly and independently associated with the development of heart failure (HF). Long-term treatment of both systolic and 
diastolic BP has been shown to reduce the risk of developing HF. HF with preserved ejection fraction is common in patients with hypertension and there is a non-linear $\mathrm{U}$-shaped relationship with increased mortality at the lower and higher end of BP control. Drugs that are used to treat hypertension also show an independent mortality benefit in HF with preserved ejection fraction (beta-blockers, ACEi/ARB). Paradoxically, once HF with reduced ejection fraction is present, patients with higher systolic BP have better outcomes. The population with HF and lower systolic BP $(<110 \mathrm{mmHg})$ are at higher risk of hospitalisation for $\mathrm{HF}$ and had more severe HF than those with systolic BP $>120 \mathrm{mmHg} .{ }^{13}$ Hypertension is associated with the development of atrial fibrillation and remains a key part of the risk assessment for anticoagulation in stroke prevention.

\section{Aldosterone and resistant hypertension}

At least $5 \%$ of patients with hypertension have adrenal aldosterone-producing adenomas, which are potentially curable with surgery. Aldosterone has adverse cardiovascular effects independent of BP. Those with resistant hypertension, hypertension in the young or those with low potassium (especially with thiazide diuretics) should be screened for primary hyperaldosteronism with plasma renin. Further investigation may include computerised tomography/magnetic resonance imaging adrenal glands followed by adrenal vein sampling or, if available, ${ }^{11} \mathrm{C}$-metomidate positron emission tomography, which has the ability to detect small adenomas $(<1 \mathrm{~cm})$ often not seen with standard imaging techniques. Treatment is with either laproscopic adrenalectomy or medical management with spironolactone, eplerenone or amiloride. The molecular basis for adrenal aldosteroneproducing adenomas has been studied with mutations found in KCNJ5, ATP1A1 and CACNA1D giving insight into distinct subtypes. ${ }^{14,15}$ Primary hyperaldosteronism should no longer be thought of as a rarely encountered condition but the diagnosis remains highly reliant upon clinical suspicion.

\section{Non-adherence to antihypertensive therapy}

Non-adherence with medication is a common problem in chronic diseases and very common in hypertension. Nonadherence is associated with higher risk of cardiovascular events and mortality and a cost to the NHS of $>\mathfrak{E} 100$ million per year. Half of patients thought to have resistant hypertension have been found to be completely or partially non-adherent. ${ }^{16}$ Measures of adherence can take the form of traditional patient interviews, pill counts, medication refill queries or admission to hospital for directly observed therapy and measurement of BP response. High-performance liquid chromatography-tandem mass spectrometry analysis of the urine gives clinicians and researchers a highly sensitive and convenient method to screen for antihypertensive drugs in blood or urine samples. These results can allow for detection of truly treatment-resistant hypertension or provide a platform for open discussion with the non-adherent patient.

\section{Salt targets: is low salt better?}

Average global consumption of salt is $10.2 \mathrm{~g} /$ day. The

Yanomamo Indian tribe of South America continue to consume an evolutionary diet containing less than $1 \mathrm{~g}$ salt per day and do not suffer from hypertension (male average BP 96/61 mmHg) or vascular disease. While there is some controversy regarding salt intake and cardiovascular disease, there is no evidence that a low salt diet increases risk of myocardial infarction or stroke. Methods of estimating and measuring dietary salt intake in clinical practice and clinical trials are often problematic. Reduction in dietary salt leads to a reduction in blood pressure ${ }^{17}$ and cardiovascular risk and is effective across all ethnicities, age groups, socio-economic classes and both genders. ${ }^{18}$

\section{New drugs in hypertension}

Despite the availability of numerous antihypertensive drugs, new drugs with improved safety profiles that have a greater effect on BP, are better targeted and better tolerated are required. Endothelin (ET) receptor antagonists and phosphodiesterase type 5 (PDE5) inhibitors for those with resistant hypertension or diabetic nephropathy provided a focus of a talk on new drugs in hypertension. ET receptor antagonists ${ }^{19}$ and PDE5 receptor antagonists ${ }^{20}$ have been shown to reduce blood pressure and reduce proteinuria and show promise as new treatments for hypertension. New drugs for hypertension are likely to be directly targeted at high-risk groups or indirectly through drug repurposing.

\section{Device therapy for hypertension}

Following the failure of the SIMPLICITY-3 trial to show benefit from renal denervation, it was unclear what would be the future of device therapy for hypertension. There are ongoing trials aiming to improve patient selection for renal denervation and improving techniques. Carotid baroreflex activation shows promise in reducing blood pressure and trials are ongoing. The ROX Coupler device (ROX Medical, San Clemente, CA, USA), which creates a conduit ateriovenous anastomosis allowing shunt of arterial blood into the venous system, is currently being evaluated.

\section{Conclusions}

Hypertension remains a global health epidemic. Conflicting evidence surrounding the optimum BP targets presents a challenge to the hypertension societies currently revising their guidelines. Emerging evidence of the superiority of spironolactone over other fourth-line antihypertensive agents is likely to feature in upcoming guidelines. Patient adherence and lifestyle interventions continue to be challenging. The emergence of single pill combinations, new antihypertensive agents and device therapy along with the results of the ongoing clinical trials in the field of hypertension will be of great interest in the near future.

\section{Conflicts of interest}

The author has no conflicts of interest to declare.

\section{References}

1 Poulter NR, Prabhakaran D, Caulfield M. Hypertension. Lancet 2015;386:801-12.

2 Elliott WJ. The economic impact of hypertension. J Clin Hypertens 2003;5:3-13. 
3 Williams B, MacDonald TM, Morant S et al. Spironolactone versus placebo, bisoprolol, and doxazosin to determine the optimal treatment for drug-resistant hypertension (PATHWAY-2): a randomised, double-blind, crossover trial. Lancet 2015;386:2059-68.

4 Group SR, Wright JT Jr, Williamson JD et al. A randomized trial of intensive versus standard blood-pressure control. N Engl J Med 2015;373:2103-16.

5 Mancia G, Grassi G. Aggressive blood pressure lowering is dangerous: the J-curve: pro side of the arguement. Hypertension 2014;63:29-36.

6 Vidal-Petiot E, Ford I, Greenlaw N et al. Cardiovascular event rates and mortality according to achieved systolic and diastolic blood pressure in patients with stable coronary artery disease: an international cohort study. Lancet 2016;388:2142-52.

7 Rapsomaniki E, Timmis A, George J et al. Blood pressure and incidence of twelve cardiovascular diseases: lifetime risks, healthy life-years lost, and age-specific associations in 1.25 million people. Lancet 2014;383:1899-911.

8 Cruickshank JM. Antihypertensive treatment and the J-curve. Cardiovasc Drugs Ther 2000;14:373-9.

9 Fagard RH, Staessen JA, Thijs L et al. On-treatment diastolic blood pressure and prognosis in systolic hypertension. Arch Intern Med 2007;167:1884-91.

10 Mancia G, Grassi G. The autonomic nervous system and hypertension. Circ Res 2014;114:1804-14.

11 Mancia G, Facchetti R, Bombelli M, Grassi G, Sega R. Long-term risk of mortality associated with selective and combined elevation in office, home, and ambulatory blood pressure. Hypertension 2006;47:846-53.

12 McManus RJ, Mant J, Bray EP et al. Telemonitoring and selfmanagement in the control of hypertension (TASMINH2): a randomised controlled trial. Lancet 2010;376:163-72.
13 Anand IS, Rector TS, Kuskowski M et al. Effect of baseline and changes in systolic blood pressure over time on the effectiveness of valsartan in the Valsartan Heart Failure Trial. Circ Heart Fail 2008;1:34-42.

14 Azizan EA, Poulsen H, Tuluc P et al. Somatic mutations in ATP1A1 and CACNA1D underlie a common subtype of adrenal hypertension. Nat Genet 2013;45:1055-60.

15 Funder JW Medicine. The genetics of primary aldosteronism. Science 2011;331:685-6.

16 Hameed MA, Tebbit L, Jacques N, Thomas M, Dasgupta I. Nonadherence to antihypertensive medication is very common among resistant hypertensives: results of a directly observed therapy clinic. J Hum Hypertens 2016;30:83-9.

17 Cappuccio FP Pro.: Reducing salt intake at population level: is it really a public health priority? Nephrol Dial Transplant 2016;31:1392-6.

18 He FJ, MacGregor GA. Salt reduction lowers cardiovascular risk: meta-analysis of outcome trials. Lancet 2011;378:380-2.

19 Kohan DE, Pritchett Y, Molitch M et al. Addition of atrasentan to renin-angiotensin system blockade reduces albuminuria in diabetic nephropathy. J Am Soc Nephrol 2011;22:763-72.

20 Oliver JJ, Hughes VE, Dear JW, Webb DJ. Clinical potential of combined organic nitrate and phosphodiesterase type 5 inhibitor in treatment-resistant hypertension. Hypertension 2010;56:62-7.

Address for correspondence: Dr Linsay McCallum, Institute of Cardiovascular and Medical Sciences, British Heart Foundation Clinical Research Centre, University of Glasgow, 126 University Place, Glasgow G12 8TA, UK.

Email: linsay.mccallum@glasgow.ac.uk

\section{NGC Natoona \\ Guideline \\ Centre}

\section{New to systematic reviewing, or need to improve your critical appraisal skills?}

Our intensive 1-day courses will introduce you to the key principles of critical appraisal and systematic reviewing, and equip you with the basic skills to put your knowledge into practice.

> Introduction to critical appraisal

> Systematic reviews and meta-analysis in action

Discount available if both courses are booked together.

'Informative and practical' Dr Rajeswari Ramaraj
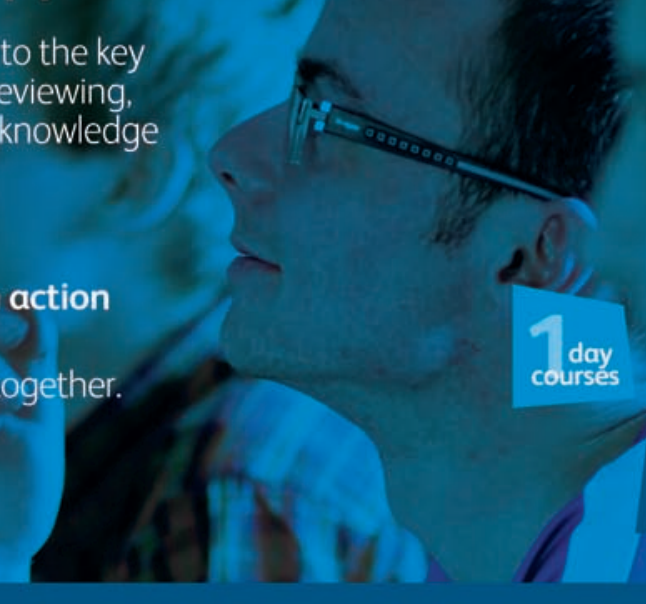